\title{
Comprensión estructural de textos escritos en estudiantes de nivel secundaria de Lima Metropolitana
}

\author{
Structural comprehension of written texts in secondary students of Lima Metropolitana
}

Compreensão estrutural de textos escritos em alunos do ensino médio na região metropolitana de Lima

Nelson Paucca Gonzales

nelfilosofia@gmail.com

https://orcid.org/0000-0001-9589-864X

Escuela de posgrado de Policía Nacional del Perú, Chorrillos-Perú

Cesar Cobos Ruiz

ccobos273@gmail.com

https://orcid.org/0000-0003-4330-8147

Universidad Nacional de Educación, Luringancho-Chosica, Perú

\section{Freddy Gamaniel Romaní Allende}

fromani_54@goutlook.com https://orcid.org/0000-0002-1054-6715

Universidad Cesar Vallejo, Lima-Perú

Rusbel Jhon Rafayle Cuadra rjrafayle@gmail.com https://orcid.org/0000-0002-2235-071X

Universidad Nacional Mayor de San Marcos, Lima-Perú

\author{
Hilarion Paucca Gonzales \\ hilarionpg@hotmail.com \\ https://orcid.org/0000-0002-1849-5174 \\ Universidad Cesar Vallejo, Lima-Perú
}

Artículo recibido 27 de septiembre 2021, arbitrado y aceptado 29 de octubre 2021 y publicado 30 de diciembre 2021

\section{RESUMEN}

Los problemas de comprensión de textos escritos en los estudiantes de los niveles de Educación Básica Regular en Perú, muestran cierto grado de deficiencia, por lo que la investigación tuvo como objetivo central, comparar la comprensión estructural de textos escritos en estudiantes de un colegio público y parroquial. Metodológicamente la investigación corresponde al método estadístico de enfoque cuantitativo, tipo básico-no experimental y de diseño causal comparativo. La información se obtuvo a través de la técnica de la encuesta y el instrumento denominado tipo de prueba. Esta última se aplicó a 130 estudiantes del grupo muestral. Finalmente, para el contraste de hipótesis se utilizó la prueba de Wilcoxon, la cual permitió concluir que no existen diferencias significativas estadísticas en la comprensión de textos escritos. Además, de las dimensiones de microestructura, macroestructura y superestructura en estudiantes de quinto grado de nivel secundaria de la ciudad de Lima - Perú.

Palabras clave: Estructuras textuales; Lingüística; Psicolingüística; Microestructura; Macroestructura; Superestructura

\section{ABSTRACT}

The comprehension problems of written texts in the students of Regular Basic Education levels in Peru, showed a certain degree of deficiency, so the main objective of the research was to compare the structural comprehension of written texts in students of a public and a parochial school. Methodologically the research corresponds to the statistical method of quantitative approach, basic type - nonexperimental and of comparative causal design. The information was obtained through the survey technique and the instrument called type of test. The latter was applied to 130 students in the sample group. Finally, for the hypotheses contrast, the Wilcoxon test was used, which would conclude that there are no statistical differences in the understanding of written texts. In addition, the dimensions of microstructure, macrostructure and superstructure in fifth-grade high school students from the city of Lima-Peru.

Key words: Textual structures; Linguistic; Psycholinguistic; Microstructure; Macrostructure; Superstructure

\section{RESUMO}

Os problemas de compreensão de textos escritos em alunos dos níveis do Ensino Fundamental Regular em nosso país apresentam certo grau de deficiência, portanto o objetivo principal da pesquisa foi comparar a compreensão estrutural de textos escritos em alunos de uma escola pública e paroquial. Metodologicamente, a pesquisa corresponde ao método estatístico de abordagem quantitativa, tipo básico - não experimental e de delineamento causal comparativo. As informações foram obtidas por meio da técnica de survey e do instrumento denominado tipo de teste. Este último foi aplicado a 130 alunos do grupo amostral. Por fim, para o contraste de hipóteses, foi utilizado o teste de Wilcoxon, o que permitiu concluir que não há diferenças estatisticamente significantes na compreensão de textos escritos. Além disso, as dimensões da microestrutura, macroestrutura e superestrutura em alunos do $5^{\circ}$ ano do ensino médio da cidade de Lima - Peru.

Palavras-chave: Estruturas textuais; Linguística; Psicolinguística; Microestrutura; Macroestrutura; Superestrutura 


\section{INTRODUCCIÓN}

La comprensión en los textos escritos en estudiantes de la Educación Básica Regular del sector público y privado de nuestro país ha venido mostrando cierta deficiencia, tal como se puede verificar en los resultados emitidos por el Programa para la Evaluación Internacional de Estudiantes (PISA, 2018), en la que el Perú ocupa el puesto 64 de un total de 77. Por tanto, se ha considerado realizar un estudio comparativo entre los estudiantes de dos instituciones de Lima Metropolitana teniendo en cuenta las proposiciones estructurales de los textos escritos. El desarrollo de la investigación tiene sus fundamentos en las afirmaciones de Van Dijk y Kintch (1983), quienes han reflexionado de manera sólida respecto a las codificaciones de los sistemas informativos de los textos escritos, enfatizando los aportes de la psicología y, más específicamente, de la lingüística, disciplinas que servirán de soporte en la construcción del marco teórico del trabajo, además, se integran en la psicolingüística. Por otra parte, se tiene en cuenta como agentes motivadores a los docentes de nivel secundaria, sin exclusión alguna, tratando de superar la visión tradicional que ha tendido a considerar a los docentes de comunicación como únicos responsables de desarrollar la comprensión lectora en los educandos. Seguir pensando de esta forma y responsabilizar solo a los docentes de comunicación es una de las causas del fracaso en los estudiantes. En ese sentido, una de las formas de revertir estos hechos es abordando la temática de manera transversal, involucrando a todos los docentes de las diferentes áreas existe la posibilidad de despertar el interés en los educandos, la pasión por la lectura y la buena redacción. En ese sentido, se debe tener en cuenta que la lectura desarrolla las habilidades de comprensión y retención de la información a partir de los textos que leen, además de la capacidad dialógica a través del uso del léxico y la capacidad de intervención por los sujetos dentro la sociedad a través de la formulación de opiniones, mostrando la consistencia de la información propia del desarrollo de la capacidad del juicio crítico. Se tiene la apreciación de que, a partir de la exigencia de los docentes, se da la posibilidad de no solo generar motivación por la lectura y la escritura sino también mejorar el uso de las reglas gramaticales y ortográficas en la redacción de los diversos tipos de textos.

Aixala (2012) Barberousse y Vargas (2019) sostienen que en principio es fundamental la pasión por la lectura para luego redactar de manera reflexiva y crítica; además, esta pasión va inculcando en los demás de manera paulatina el que otros también se involucren en la práctica del libre pensamiento bajo las concepciones de un ciudadano demócrata. Si bien es cierto, para los ciudadanos es de suma importancia leer y escribir a fin de ser hombres informados, además de practicar la democracia en beneficio del bien común, para que puedan asumir estas responsabilidades los individuos deben ser orientados oportunamente desde el momento en que se insertan en la escuela, los estudiantes deben ser asistidos por el equipo de la casa y los padres de familia a través del ejemplo. Esto conlleva que también los padres deben leer necesariamente, puesto que solo así los estudiantes podrán insertarse en el mundo de la lectura, además de ir formando hábitos que se repliquen en su entorno.

Por otra parte, el estudio del Instituto de Estadística de la UNESCO refiere que niños y 
adolescentes escolares en América Latina no han logrado las competencias básicas concernientes a la compresión de lectura. Análisis (2017) muestra que el $36 \%$ de estudiantes en América Latina están inmersos en este déficit. Dentro de esta, se encuentra involucrado el Perú por presentar un alto déficit en la comprensión lectora y escritura.

En referencia a los acápites mencionados, compete de un análisis exhaustivo el presente trabajo. Es de notarse, la falta de hábitos de lectura en la sociedad en general, además de constatarse la poca motivación por parte de los agentes de las instituciones educativas, a ello se suma la familia, puesto que no aporta como espera el proceso de formación en lo que concierne al desarrollo de la lectura, como se dijo, esta obedece a diversos factores; como la pobreza, la misma que no les permite acceder a la compra de libros, textos o materiales de lectura. Por otra parte, los padres de familia, especialmente aquellos de hogares con limitaciones económicas no priorizan la adquisición de los materiales y no le da mayor importancia a la lectura, justificando que la mayor parte de su tiempo los dedican al trabajo. Por ende, salen a trabajar temprano y retornan a casa tarde, muchas veces los hijos son dejados durmiendo por los padres y al retornar también los encuentran durmiendo, de esta se deduce que los estudiantes se encuentran en estado de desamparo, también, cabe sostener que los estudiantes viven solos y no hay nadie que se puede hacer cargo de su formación, como lo señala Guzmán (2017), la cual refiere que durante un año las personas no suelen leer y si leen no terminan de leer ni siquiera un libro al año, por este motivo no se logra desarrollar la comprensión lectora (Almandoz, et al, 2019). Frente a estos resultados la tarea del sistema educativo es justamente buscar algunas estrategias con la finalidad de revertir la situación caótica. Se sabe que un país sin hábitos de lectura y escritura está sentenciado a la ignorancia y la miseria de por vida. Teniendo en cuenta esta realidad, se ha planteado la siguiente pregunta $¿ D e$ qué manera se muestra el nivel de comprensión estructural de textos escritos en los estudiantes de quinto grado de educación secundaria de dos instituciones educativas de la ciudad de Lima? Y como objetivo central; comparar la compresión estructural de textos escritos en los estudiantes de quinto grado de educación secundaria de dos instituciones educativas de la ciudad de Lima.

En función al objetivo central cabe esbozar que la realidad de la población a nivel local es de una u otra forma controversial, primero se tiene en cuenta que los estudiantes de quinto grado de nivel secundaria quienes cursan el último año de su vida colegial muestran cierto grado de dificultad respecto a la comprensión de los textos que leen. En ese sentido cabe sostener que la educación tiene sus principios formidables en el hogar, aquello implica que los padres de deben asumir su rol en el acompañamiento de los aprendices, con la finalidad de no someterlo al desamparo, en caso de no ser así, con toda probabilidad los estudiantes presentarán dificultades en el ejercicio de la educación superior como también en la praxis laboral. Para superar esta dificultad, el estudiante o cualquier otro individuo debe tener un propósito, partiendo del título hasta sumergirse propiamente en la lectura, tarea que debe ser reforzada por los docentes en las escuelas. Una de las formas de motivar la pasión por la lectura de parte del docente es justamente llevando consigo un libro, ya que esta genera un acercamiento del 
sujeto con el libro, por otra parte, es necesario colocar diversos textos dentro de las aulas donde se desarrollan las clases con la finalidad de entrever la exploración por parte de los aprendices. En consecuencia, es necesario que el docente realice comentarios de manera introductoria para que los lectores se involucren en ella. Estas y otras estrategias generadas por el docente deben aplicarse desde que los niños se ponen en contacto con la escuela.

\section{Comprensión de textos escritos}

La investigación ha tenido como variable general la comprensión de textos escrito. Para la elucidación de la variable se ha tenido en cuenta las propuestas de Velásquez, et al (2008), quien concibe que el texto es un conjunto integrado de oraciones y esta a su vez expresa un significado global. Se han considerado también los argumentos sostenidos en torno a la concepción lingüística sostenido por Van Dijk (1980), quien establece con claridad una diferencia en lo que respecta a las terminologías texto y discurso; refiriéndose a este último, sostiene que "es una unidad observacional, es decir, la unidad que interpretamos al ver o escuchar una emisión" (p. 20). Por tanto, el discurso surge a partir de la observación, la que a su vez será interpretada por el observador; por otra parte, en lo que concierne al texto, sostiene que este es un derivado de una disyuntiva del vocablo holandés y alemán, en ese sentido el texto necesariamente posee un significado. Se tienen en cuenta que los textos escritos son necesariamente expresados a través de las oraciones, las mismas que son integrados en párrafos con la finalidad de expresar un mensaje de carácter global. Por otro lado, Reguera (2016) y Niño (2019) sostienen que el discurso es uno de los instrumentos que permite a las personas entenderse de manera pertinente dentro de una determinada sociedad, o durante el proceso de interacción comunicativa. Si bien es cierto, desde la concepción de Van Dijk el discurso es una unidad observacional que naturalmente estaría relacionado con los estudiantes, en el presente caso, la unidad de análisis es justamente el grupo muestral al que se ha aplicado los instrumentos para recoger la información; sin embargo, desde la concepción de Reguera el discurso es un instrumento de análisis que permite necesariamente entender las prácticas discursivas realizadas por las personas. Teniendo en cuenta las concepciones de las terminologías, es necesario que los docentes y los estudiantes diferencien lo que es un discurso y un texto.

En síntesis, si bien es cierto, el discurso se puede aprender a través de la observación y la escucha, esta pareciera ser sencillo, pero lo que se debe tener en cuenta es a quiénes se debe observar y escuchar. La observación y la escucha siempre debe estar relacionadas con lo que se considera culto, pero debemos tener en cuenta que los estudiantes no siempre tienen la misma inquietud que los adultos. Ellos observan y escuchan de acuerdo a su interés específico del momento, y creemos que es tarea de la escuela contribuir a que el estudiante dilucide la conveniencia, ventaja o importancia mayor de un tipo de material de lectura sobre otro. Para afrontar estas situaciones que se van presentando en la vida cotidiana de los estudiantes, los decentes necesariamente deben guiarlo en su inserción al mundo de la lectura escrita. Hombre que no lee estará condenado a la sumisión de los protagonistas. Se debe tener en cuenta que el hombre que ejerce el discurso es necesariamente un hombre culto, dedicado a la lectura de manera constante. 
Según Van Dijk (1992) la capacidad de discernir las diversas informaciones que nos puede brindar el texto debe ser entendida a partir de los significados globales, los mismas que posibilitan determinar el tema central. El logro de los objetivos tiene su punto de partida en el análisis de las ideas de cada párrafo del texto. Matéus (2007) por su parte, presenta un análisis desde la perspectiva psicolingüística relacionando con la comprensión de textos, argumentando que esta debe partir de la construcción, la misma que debe ser analizada con miras de la reestructuración. De esta manera es posible sistematizar las informaciones con la finalidad de convertirlo en texto escrito y este a su vez podría convertirse en uno nueva versión del texto original, tanto en su forma como en su significado (Goodman, 1971, citado en Carrel et al (1988). Este proceso de manera repetida quedaría almacenado en el cerebro del lector y permitiría encontrar diferentes significados del texto de manera interiorizada. Cabe notarse que el procesamiento de la información depende únicamente del lector; por tanto, el problema no radica en la existencia o la construcción de los textos escritos sino más bien en las personas que leen para construir una nueva información. En ese sentido, la función de los maestros consiste en proveer herramientas a los estudiantes a fin de generar una alta preparación en los hábitos que comprometen la comprensión de textos escritos.

Van Dijk y Kintch (1983) enfatizan que el texto se constituye en microestructura, macroestructura y superestructura. Esta última es el mayor soporte en función a los dos anteriores, y cada una de estas a su vez guarda relación con la disciplina lingüística. La microestructura del texto guarda relación con el aspecto semántico de la lingüística, mientras que la macroestructura se vincula con la sintaxis y la superestructura con la pragmática. La propuesta de los autores tiene relevancia, y debería ser enfatizado por los docentes en el proceso de enseñanza y aprendizaje. Es necesario que los estudiantes tengan en cuenta la constitución del texto y la interrelación con la lingüística. Se ha podido notar una cierta tendencia en los docentes a exigir a sus estudiantes leer sin ninguna objetividad. En ese sentido, lo que conviene es hacer notar que un texto está constituido por la combinación interrelacionada de vocales y consonantes, las mismas que se evidencian a través de la palabra, aspecto que correspondería abordar en la parte analítica desde el enfoque microestructura. Luego, las palabras se integran para componer oraciones y párrafos, al cual corresponde el análisis del enfoque de macroestructura. Finalmente, los párrafos también se interrelacionan y conforman cuentos, narraciones, novelas, historias, etc., y estas dependen de la intención de los autores, parte completa y compleja que corresponde abordar en el análisis del enfoque superestructura; estos enfoques se relacionan con la disciplina lingüística microestructura - semántica, macroestructura - sintaxis y superestructura - pragmática. Aun cuando estas temáticas son poco desarrolladas por los docentes, tener en cuenta permitiría superar el problema de la comprensión y generaría un hábito más eficaz por la lectura y los diversos tipos de textos escritos; además, induciría a los estudiantes al razonamiento inductivo y deductivo durante el proceso de su formación, generando mayores expectativas en su vida cotidiana.

Del mismo modo, Sánchez (1989), Fuenmayor, Villasmil y Rincón (2007), Pallares (2017) y 
Matéus (2007) han realizado un análisis acerca de la propuesta de Kintch y Van Dijk en torno a la relación del texto y la disciplina lingüística. Respecto a la relación entre microestructura y la parte semántica resalta la construcción de las proposiciones, oraciones y párrafos. Estas a su vez guarden coherencia y cohesión en el texto. En tal sentido, el texto evidencia claridad y facilita a los lectores identificar el título, las ideas centrales y el significado de los diversos párrafos. La macroestructura textual y la parte sintáctica de la disciplina lingüística enfatiza la concepción de la parte teórica teniendo en cuenta la aplicación de las macroreglas con la finalidad de garantizar la organización de la información textual. Respecto a la macroestructura y la parte pragmática, sostienen que esta permite la determinación de las ideas centrales y a partir de ella se puede generalizar el contenido del texto. En este proceso interviene el ejercicio de los procesos mentales en función a la aplicación de las macroreglas.

\section{METODOLOGÍA}

El presente manuscrito metodológicamente cumple las características estructurales de lo que exige la ciencia. Por otra parte, se ha tenido en cuenta el uso del método descriptivo hermenéutico, puesto que esta ha permitido describir desde la descripción problemática hasta la obtención de los resultados, en cada proceso se ha analizado apelando a la hermenéutica, además se ha tenido en cuenta el método estadístico, ya que esta ha permitido disipar las analogías comparativas. En consecuencia, el uso del método se relaciona con los sostenido por Paucca, Rafayle y Andrade (2020) "el método científico es un procedimiento para tratar un conjunto de problemas" (p. 92). Como es de notarse, el desarrollo de una investigación requiere el uso de métodos cualitativos y cuantitativos tal es caso de la hermenéutica que permite analizar la parte teórica mientras que la estadística permite comparar los resultados.

En lo que concierne al enfoque se ha tenido en cuenta lo cualitativo de diseño no experimental, puesto que la variable y las dimensiones propuestos han sido medidas, por su parte (Hernández, Fernández y Baptista, 2013; Vara, 2012) sostienen que el presente enfoquetiene como objetivo principal recoger la información con la finalidad de realizar la contrastación de la hipótesis. El diseño se ha caracterizado por la facilitación en lo que concierne a la determinación de los grupos muestrales con la finalidad de formular la problemática a investigar Bisquerra (2019). La población estuvo conformada por los estudiantes de nivel secundaria de ambas instituciones educativas y la muestra conformada por un total 130 estudiantes de quinto grado de nivel secundaria conformado por ambas instituciones, en ese Barboza (2018) sostiene que la población es el conjunto conformado por personas, mientras que la muestra es una parte proporcional del total de la población que ha sido determinado por el equipo investigador. La muestra se ha seleccionado a través del muestreo no probabilístico por conveniencia, la técnica usada es la encuesta y el instrumento ha sido el cuestionario de 21 ítems de tipo dicotómico de alternativa múltiples, este último se validó a través de los jueces expertos con una puntuación de $(0,92)$ y esta se determinó recurriendo al estadístico Alfa de Cronbach. Finalmente, el procesamiento de la data se usó Microsoft Excel y el software Paquete Estadístico para las Ciencias Sociales (SPSS), versión 25. Para la obtención de los resultados siguió dos fases: en la primera se tuvo en cuenta la estadística 
descriptiva; los resultados de la segunda parte de la investigación, obedecen a la estadística inferencial.

\section{RESULTADOS}

Los resultados del presente acápite corresponden a la estadística descriptiva, la misma que ha permitido responder los objetivos planteados. En la Figura 1 se describe la diferencia estadística de la variable comprensión de textos escritos entre los estudiantes de una institución parroquial y nacional, en ese sentido se puede notar que los estudiantes San Salvador-Parroquial oscila entre las puntuaciones en nivel proceso $(32=$ $49.2 \%)$ y satisfactorio $(30=46.2 \%)$ mientras que los estudiantes de San Antonio-Nacional oscilan entre las puntuaciones en el nivel en proceso $(31=47.7 \%)$ y satisfactorio $(34=52.3 \%)$. En conclusión, los estudiantes se encuentran en similares condiciones en lo que refiere a los niveles de comprensión de textos escritos entre los estudiantes de ambas instituciones.

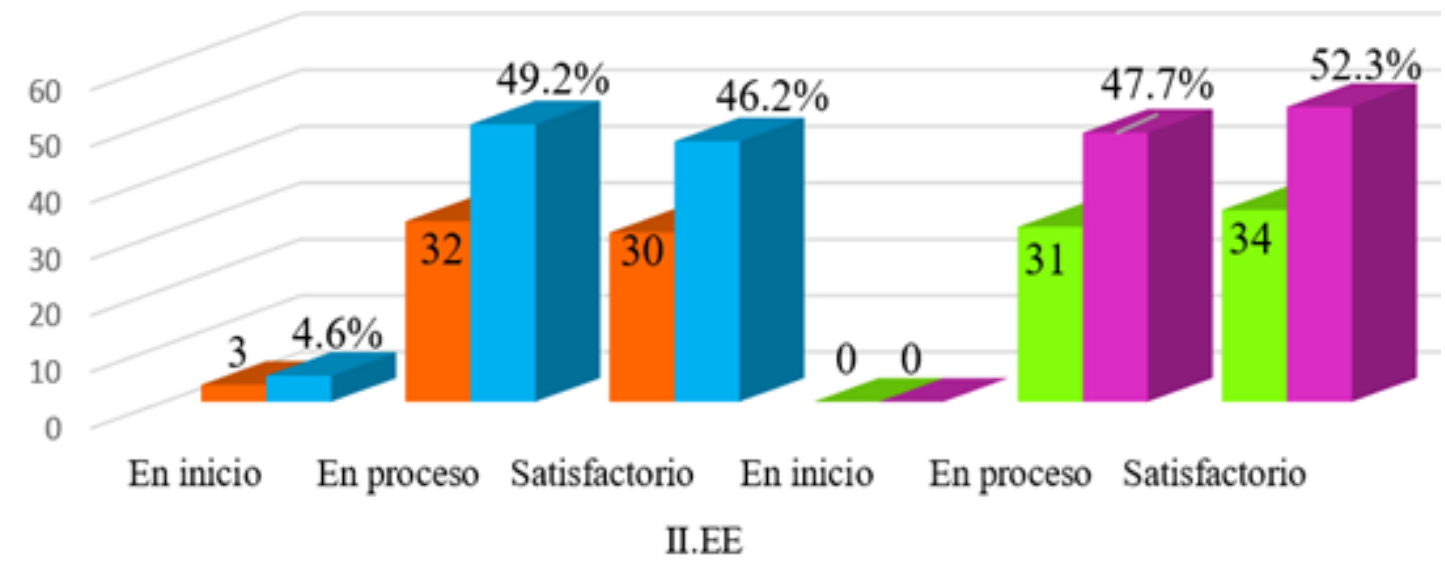

घ San Salvador $\quad$ San Antonio

Figura 1. Comparación del objetivo general entre la IE Parroquial y Estatal.

Nota. La figura muestra la parte comparativa de la escala vigesimal y los niveles en la que se encuentran los estudiantes respecto al objetivo general.

En la Figura 2 se describe la diferencia estadística de la dimensión nivel microestructura de comprensión de textos escritos entre los estudiantes de una institución parroquial y nacional, en ese sentido se puede notar que los estudiantes San Salvador-Parroquial oscila entre las puntuaciones en nivel satisfactorio $(48=73.8 \%)$ mientras que los estudiantes de San Antonio-Nacional oscilan entre las puntuaciones en el nivel satisfactorio (44 $=67.7 \%)$. En conclusión, se sostiene que en los estudiantes de ambas instituciones predomina el desarrollo del nivel microestructura de comprensión de textos escritos. 


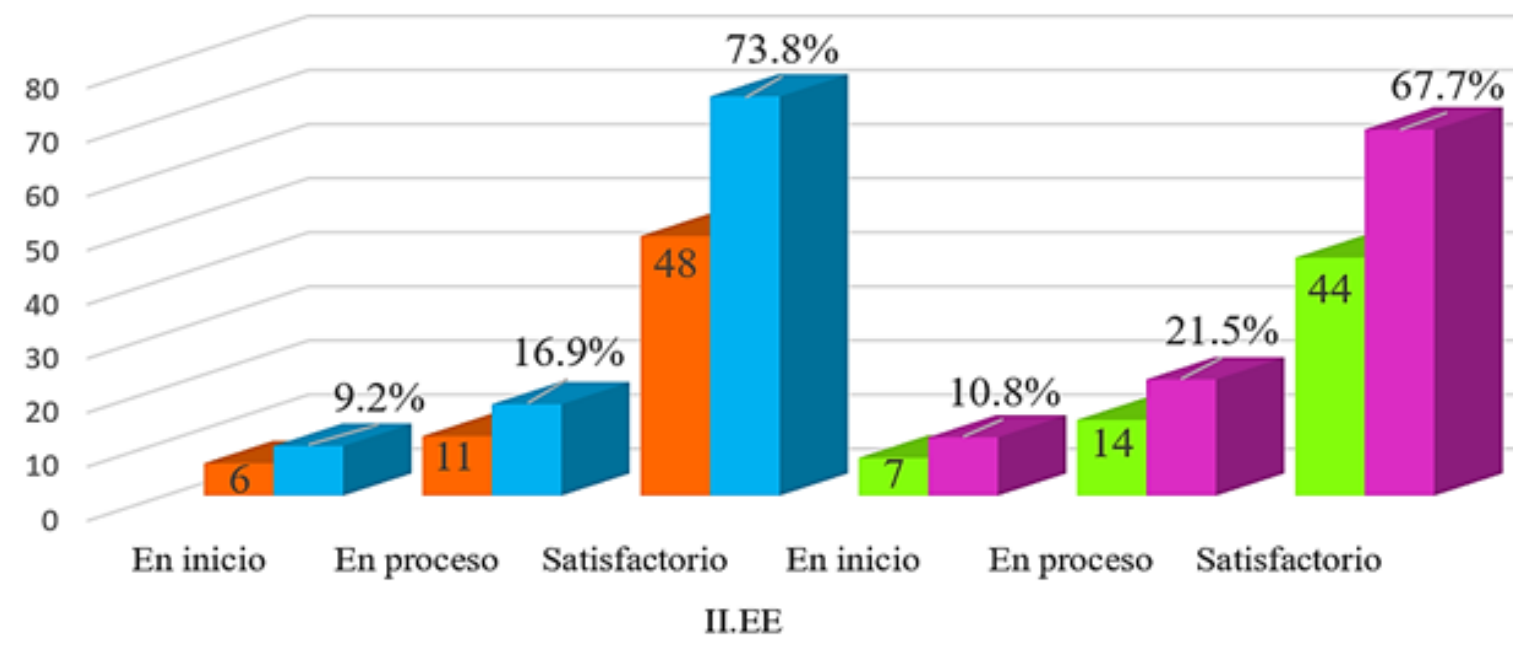

\section{a San Salvador $\quad$ San Antonio}

Figura 2. Comparación de la dimensión microestructura.

Nota. En la figura se observa la escala vigesimal de la dimensión microestructura y el nivel de ubicación de los estudiantes de ambas instituciones educativas.

La Figura 3 muestra la descripción de mientras que los estudiantes de San Antoniola diferencia estadística de la dimensión Nacional oscilan entre las puntuaciones en el nivel macroestructura de comprensión de textos escritos entre los estudiantes de una institución parroquial y nacional, en ese sentido se puede notar que los estudiantes San Salvador-Parroquial oscila entre satisfactorio $(45=69.2 \%)$. En conclusión, se sostiene que en los estudiantes de ambas instituciones predomina el desarrollo del nivel macroestructura de comprensión de textos escritos. las puntuaciones en nivel satisfactorio $(44=67.7 \%)$

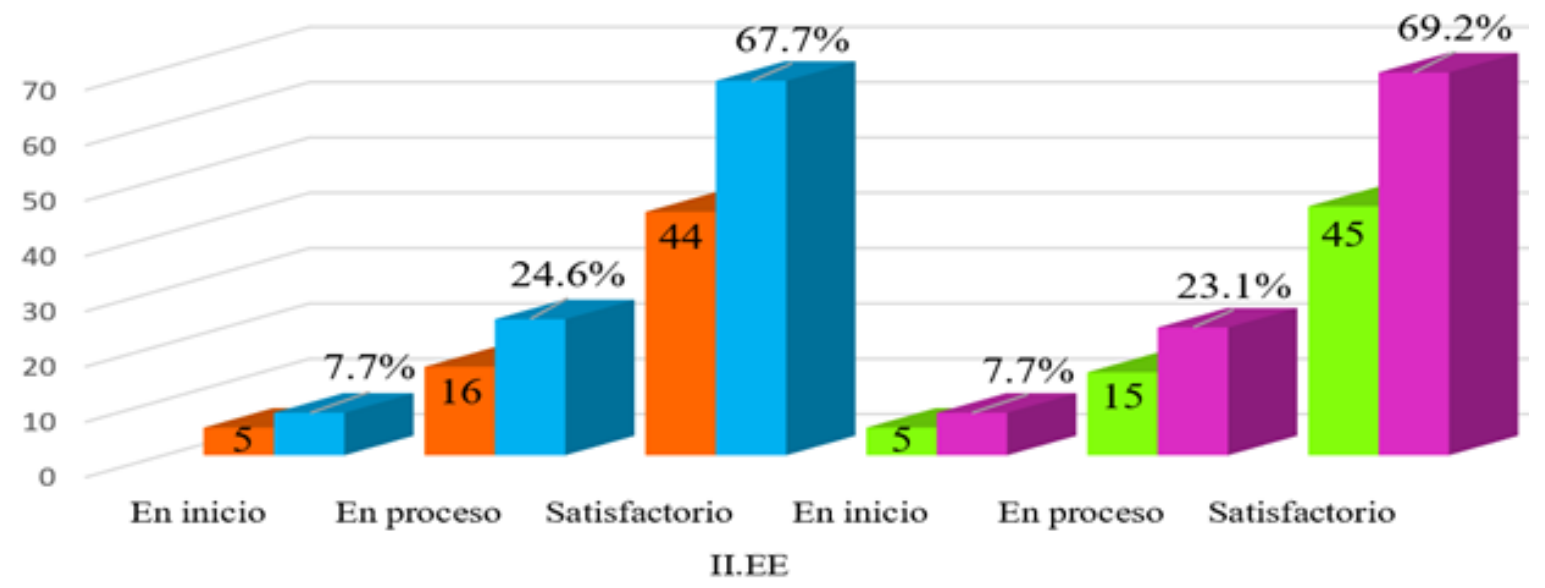

- San Salvador

- San Antonio

Figura 3. Comparación de la dimensión macroestructura.

Nota. En la figura se observa la escala vigesimal de la dimensión macroestructura y el nivel de ubicación de los estudiantes de ambas instituciones educativas. 
De la Figura 4 se colige la descripción de la diferencia estadística de la dimensión superestructura de comprensión de textos escritos entre los estudiantes de una institución parroquial y nacional, en ese sentido se puede notar que los estudiantes San Salvador-Parroquial oscila entre las puntuaciones en nivel en proceso $(34=52.3 \%)$ mientras que los estudiantes de San AntonioNacional oscilan entre las puntuaciones en el nivel en proceso $(30=46.2 \%)$. En conclusión, se sostiene que en los estudiantes de ambas instituciones tienen dificultad el desarrollo del nivel superestructura de comprensión de textos escritos.

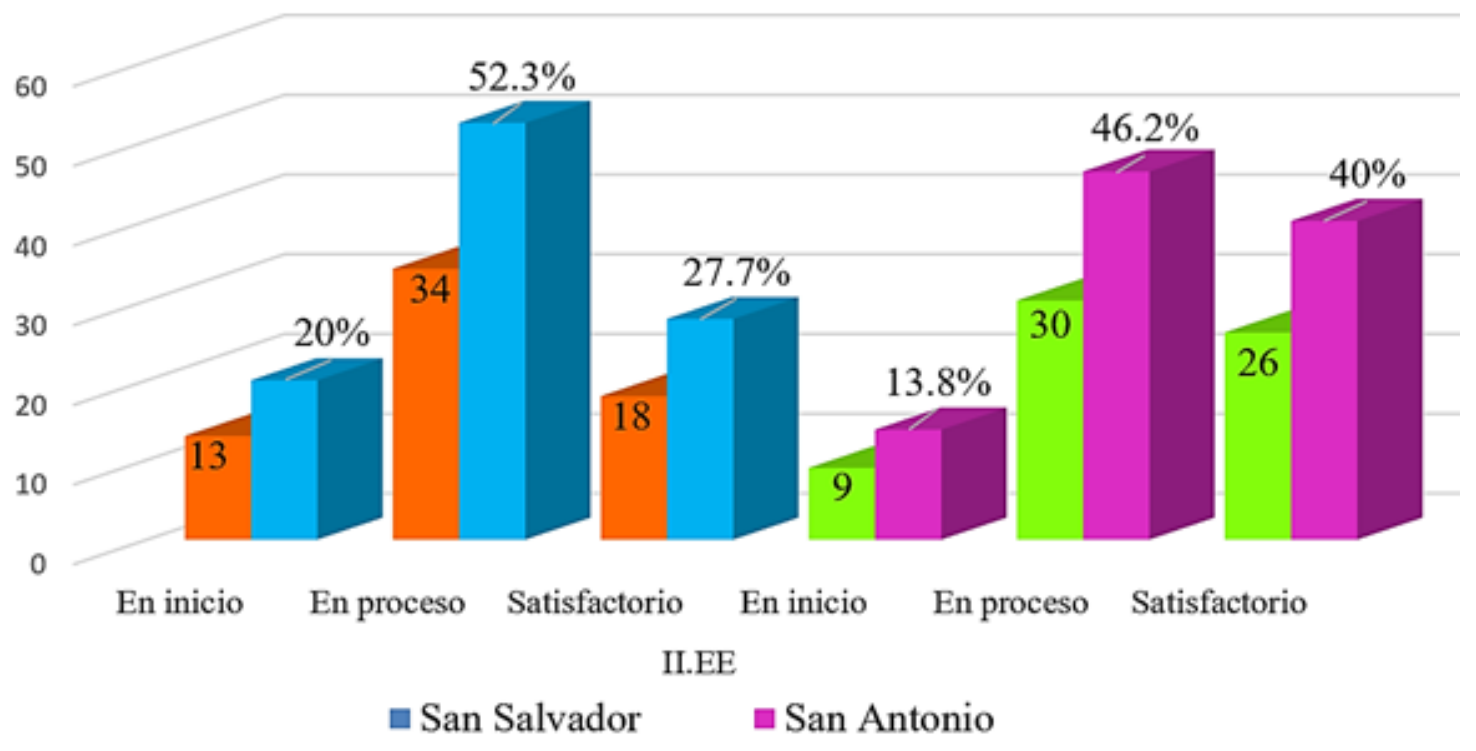

Figura 4. Comparación del objetivo específico superestructura.

Nota. En la figura se observa la escala vigesimal de la dimensión superestructura y el nivel de ubicación de los estudiantes de ambas instituciones educativas.

En la Tabla 1, según la prueba de rangos con signo de Wilcoxon, se observa que el valor de $\mathrm{Z}$ $=-1,073$ y la significancia asintótica (bilateral) es $(0.283>0.05)$, en referencia a los resultados se acepta la hipótesis nula y se concluye estadísticamente que no existen diferencias significativas en la comprensión de textos escritos en estudiantes de quinto grado de Educación Básica Regular de una institución parroquial y estatal de la ciudad de Lima durante el año 2019. 
Tabla 1. Prueba de hipótesis general a través de prueba de rangos con signo de Wilcoxon.

\begin{tabular}{lrr}
\hline & Competencia de textos escritos entre IE Estatal y Parroquial \\
\hline$Z$ & $-1,073$ &, 283 \\
Sig. asintótica (bilateral) &, 283 \\
\hline
\end{tabular}

En la Tabla 2, según la prueba de rangos con signo de Wilcoxon, se observa que el valor de $\mathrm{Z}$ $=-0,549$ y la significancia asintótica (bilateral) es $(0,583>0,05)$, en referencia a los resultados se acepta la hipótesis nula y se concluye estadísticamente que no existen diferencias significativas en la dimensión microestructura de comprensión de textos escritos en estudiantes de quinto grado de Educación Básica Regular de una institución parroquial y estatal de la ciudad de Lima durante el año 2019.

Tabla 2. Prueba de hipótesis dimensión Microestructura comprensión de textos escritos.

\section{D1_ Microestructura entre IE Estatal y Parroquial}

Z

En la Tabla 3, según la prueba de rangos con signo de Wilcoxon, se observa que el valor de $\mathrm{Z}=$ $-1,00$ y la significancia asintótica (bilateral) es $(0,920$ $>0,05)$, en referencia a los resultados se acepta la hipótesis nula y se concluye estadísticamente que no existen diferencias significativas en la dimensión macroestructura de comprensión de textos escritos en estudiantes de quinto grado de Educación Básica Regular de una institución parroquial y estatal de la ciudad de Lima durante el año 2019.

Tabla 3. Hipótesis dimensión Macroestructura comprensión de textos escritos.

D2_ Macroestructura entre IE Estatal y Parroquial

Z

En la Tabla 3, según la prueba de rangos con signo de Wilcoxon, se observa que el valor de $\mathrm{Z}$ $=-1,372$ y la significancia asintótica (bilateral) es $(0,170>0,05)$, en referencia a los resultados se acepta la hipótesis nula y se concluye estadísticamente que no existen diferencias significativas en la dimensión superestructura de comprensión de textos escritos en estudiantes de quinto grado de Educación Básica Regular de una institución parroquial y estatal de la ciudad de Lima durante el año 2019. 
Tabla 4. Hipótesis dimensión Superestructura comprensión de textos escritos.

D3_Superestructura entre IE Estatal y Parroquial

Z

\section{Discusión}

En lo que concierne a la parte teórica, de la variable comprensión de textos escritos se ha tenido en cuenta la propuesta de Van Dijk (1980) quien propone que los significados de los textos escritos se deben reconocer de manera global, puesto que este permite determinar el tema central del texto; por otra parte, el autor hace notar que la terminología lingüística es diferente respecto al texto y el discurso.

Por su parte, Pallares (2017) también realizaron un minucioso seguimiento a los enunciados hechas por Van Dijk en lo concierne a la estructura de un texto. De la misma forma, se tiene en cuenta la propuesta de Matéus (2007) quien sostiene, desde el enfoque psicolingüístico, que el entendimiento de las informaciones es posible construirlo en función a los textos escritos existentes. El autor sostiene que la información obtenida es procesada en la mente a fin de ser expresado como una respuesta relevante. En ese sentido, los docentes deben generar una mayor apertura en los estudiantes el procesamiento de la información a partir de lo que leen, el logro de sus entendimientos debe ser constado a través de la emisión de las respuestas de manera autónoma con argumentos sólidos y fundados del desarrollo de los textos escritos a partir de los contenidos de las lecturas. En consecuencia, los estudiantes deben tener claro el ¿Por qué de su desarrollo de un texto escrito? Estas reflexiones se fundamentan en las propuestas de Velásquez, et al (2008), quien afirma que conceptualmente el texto es un conjunto de oraciones que a su vez tienen un significado global.

En lo que concierne al instrumento, cabe mencionar que este ha sido adaptado teniendo en cuenta a Pallares (2017). La adaptación ha sido validada con la finalidad de contextualizar y potenciar su aplicabilidad a través de los jueces expertos, quienes le asignaron una puntuación de (0.92) y esta se determinó recurriendo al estadístico Alfa de Cronbach. A consecuencia de la validación también se verificó la confiabilidad a través del grupo piloto, del cual se obtuvo una puntuación de (0.86) a través del estadístico Kuder - Richardson. La validación y confiabilidad del instrumento se ciñe a las teorías de Kintch y Van Dijk (1983), la misma que ha permitido dimensionar la variable general en tres dimensiones.

Respecto a los resultados, cabe resaltar que se ha trabajado en dos momentos. La primera obedece a la estadística descriptiva, lo cual nos ha permitido responder los objetivos planteados en la investigación a través de la comparación del nivel de comprensión de textos escritos entre los estudiantes de una institución Parroquial y Estatal.

En cuanto al objetivo general comprensión de textos escritos, se llegó a la siguiente conclusión; los estudiantes se encuentran en similares condiciones en lo que refiere a los niveles de comprensión de textos escritos entre los estudiantes de ambas instituciones. Referente a la dimensión 
microestructura, se llegó a la siguiente conclusión se sostieneque en los estudiantes de ambas instituciones predomina el desarrollo del nivel microestructura de comprensión de textos escritos. Respecto a la dimensión macroestructura, se llegó a la siguiente conclusión se sostiene que en los estudiantes de ambas instituciones predomina el desarrollo del nivel macroestructura de comprensión de textos escritos. Y, por último, respecto a la dimensión superestructura se llegó a la siguiente conclusión se sostiene que en los estudiantes de ambas instituciones tienen dificultad el desarrollo del nivel superestructura de comprensión de textos escritos.

El desarrollo de prueba de hipótesis obedeció a la estadística inferencial, para el cual se ha tenido en cuenta lo sostenido por Triola (2009); Bernardino (2012); y Bardales (2010), quien afirman que, cuando la muestra es mayor a 50 sujetos, se debe aplicar el estadístico Kolmogorov-Smirnov a fin de mostrar la prueba de normalidad y determinar el tipo de estadístico para probar la hipótesis planteada. En ese sentido, la prueba de normalidad es $(0,000<$ $0,05)$ indica que la distribución no es normal, por lo que se consideró al estadístico Wilcoxon para la determinación de la hipótesis.

En última instancia, la segunda parte de los resultados de la investigación obedece a la estadística inferencial, a través del cual se procesó usando el software SPSS versión 25 y la estadística no paramétrica Wilcoxon, tal como se evidencia en la tabla 1, en la cual se ha evidenciado el valor de significancia $(0,283>0,05)$ correspondiente a la hipótesis general. De la misma forma los resultados de las dimensiones microestructura $(0.583>0.05)$, macroestructura $(0.920>0.05)$ y superestructura $(0.170>0.05)$ también son mayores a $(0.05)$, por lo que se sostiene que estadísticamente no existen diferencias significativas en la comprensión de textos escritos en estudiantes de quinto grado de Educación Básica Regular de una institución estatal y parroquial de la ciudad de Lima durante el año 2019. En ese sentido, se rechazó la hipótesis planteada por el investigador y se aceptó la hipótesis nula.

\section{CONCLUSIONES}

En cuanto al objetivo general comprensión de textos escritos, se llegó a la siguiente conclusión; los estudiantes se encuentran en similares condiciones en lo que refiere a los niveles de comprensión de textos escritos entre los estudiantes de ambas instituciones.

Referente a la dimensión microestructura, se concluye; que en los estudiantes de ambas instituciones predomina el desarrollo del nivel microestructura de comprensión de textos escritos.

Respecto a la dimensión macroestructura, se concluye; que en los estudiantes de ambas instituciones predomina el desarrollo del nivel macroestructura de comprensión de textos escritos.

$\mathrm{Y}$, por último, respecto a la dimensión superestructura se concluye; que en los estudiantes de ambas instituciones tienen dificultad el desarrollo del nivel superestructura de comprensión de textos escritos.

En lo que concierne a la hipótesis general se concluye estadísticamente que no existen diferencias significativas en la comprensión de textos escritos en estudiantes de quinto grado de Educación Básica Regular de una institución parroquial y estatal de la ciudad de Lima durante el año 2019. 
En lo que refiere a la primera hipótesis especifica se concluye estadísticamente que no existen diferencias significativas en la dimensión microestructura de comprensión de textos escritos en estudiantes de quinto grado de Educación Básica Regular de una institución parroquial y estatal de la ciudad de Lima durante el año 2019.

En lo que refiere a la segunda hipótesis especifica se concluye estadísticamente que no existen diferencias significativas en la dimensión macroestructura de comprensión de textos escritos en estudiantes de quinto grado de Educación Básica Regular de una institución parroquial y estatal de la ciudad de Lima durante el año 2019.

Finalmente, respecto a la tercera hipótesis especifica se concluye estadísticamente que no existen diferencias significativas en la dimensión superestructura de comprensión de textos escritos en estudiantes de quinto grado de Educación Básica Regular de una institución parroquial y estatal de la ciudad de Lima durante el año 2019.

\section{REFERENCIAS}

Aixala, E. (2012). Leer es un derecho. Recursos para animación a la lectura. Montevideo: Ministerio de Educación y Cultura. http://studylib.es/ doc/5556826/leer-es-underecho---bibliotecaddp

Almandoz, P., Garcén, M., Ferreri, E., Barochiner, E., Delmas, A. y Chan, D. (2019). Niveles de comprensión lectora de textos científicos en estudiantes de ingeniería. RAES, 11(19), pp. 7895. http://www.revistaraes.net/revistas/raes19_ art6.pdf

Barberousse, P y Vargas, M. (2019). Animación a la lectura y escritura en la Escuela Finca Guararí: Una experiencia lúdico-creativa desde el proyecto "Construyendo una propuesta de implementación" del Programa Maestros Comunitarios. Revista electrónica Educare, 23
(2), 1-15. DOI: http://dx.doi.org/10.15359/ ree.23-2.7

Barboza, P. (2018). Propuesta metodológica basada en el modelo de Walter Kintsch y Teun Van Dijk para mejorar los niveles de comprensión lectora en los estudiantes del $2^{\circ}$ año de educación secundaria de la institución educativa nacional "Luis Negreiros Vega-Pátapo" (tesis de maestría). Universidad Nacional Pedro Ruiz Gallo, Perú

Bardales, C. (2010). Metodología de investigación. Pearson

Bernardino, C. (2012). Estadística y muestreo. (10 edición). ECOE

Bisquerra, R. (2019). Metodología de la investigación educativa. ( $6^{\text {a }}$ edición). La Muralla

Carrel, P. Devine, J. \& Eskey, D. (edits.) 1988. Interactive Approaches to Second Language Reading. CUP

Fuenmayor, G., Villasmil, Y. y Rincón, M. (2007). Construcción de la microestructura y macroestructura semántica en textos expositivos producidos por estudiantes universitarios de luz. Letras. http://web.b.ebscohost.com/ehost/ $\mathrm{pdfviewer} / \mathrm{pdf}$ viewer? $\mathrm{vid}=4 \&$ sid $=0 \mathrm{a} 93629 \mathrm{e}$ 4a95-42e9-a31e-8ba4c4838361\%40pdc-vsessmgr04

Guzmán, I. (12 de abril del 2017). Peruanos leen en promedio menos de un libro al año. https:// diariocorreo.pe/cultura/peruanos-leen-enpromedio-menos-de-un-libro-al-ano-742938/

Hernández, R.; Fernández, C. y Baptista, P (2013). Metodología de la investigación (sexta edición). Mc Graw-Hill

Matéus, G. (2007). Psicología de la comprensión textual y control de la comprensión: revisión de conceptos. Segunda época. http://www.scielo. org.co/pdf/folios/n26/n26a04.pdf

Pallares, I. (2017). El modelo propuesto por Van Dijk y Kintsch y la comprensión lectora de los estudiantes de noveno grado del instituto agrícola de Charta en el municipio de Charta en el departamento de Santander - Colombia 2014 (tesis de maestría). http://repositorio.uwiener. edu.pe/handle/123456789/596 
PISA. (2018). Programa para la Evaluación Internacional de los Estudiantes. https://www. observatoriodelainfancia.es/ficherosoia/ documentos/5943_d_InformePISA2018Espana1.pdf

Paucca, H. (2019). Comprensión de textos escritos de estudiantes del sétimo ciclo de una institución educativa parroquial y una estatal de Lima, 2019. (Tesis de licenciatura). http://repositorio.ucv. edu.pe/bitstream/handle/UCV/41473/Paucca GH.pdf? sequence $=1$ \&isAllowed $=y$

Paucca, N., Rafayle, J. y Andrade, J. (2020). Análisis axiológico de la construcción y validación de un instrumento documental edumétrico. Puriq, 2 (2), 1-15. DOI: https://doi.org/10.37073/ puriq.2.2.81

Reguera, A. (2016). Metodología de la investigación lingüística. (2 $2^{\mathrm{da}}$ edición). Argentina: Brujas

Sánchez, E. (1989). Procedimientos para instruir en la comprensión de textos. Recuperado de file://C:/Users/USER/Downloads/00752_19. pdf
Triola, M. (2009). Estadística. Pearson Educación

Van Dijk, T. (1980). Estructuras y funciones del discurso. Siglo veintiuno editores

Van Dijk, T. (1980). Texto y contexto. Cátedra

Van Dijk, T. (1992). La ciencia del texto Un enfoque interdisciplinario. Paidós

Van Dijk, T. y Kintsch, W. (1983). Estrategias de comprensión del discurso. Estados Unidos: New York: Academic Press. https://www.tadkiroatun. education/wp-content/uploads/2017/02/TeunA-van-Dijk-Walter-Kintsch-Strategies-ofDiscourse-Comprehension.pdf

Vara, A. (2012). 7 pasos para una tesis exitosa. Universidad San Martín de Porras

Velásquez, M., Cornejo, C y Roco, A. (2008). Evaluación de la competencia lectora en estudiantes de primer año de carreras del área humanista y carreras del área de la salud en tres universidades del consejo de rectores. Estud. Pedagóg. https://scielo.conicyt.cl/pdf/estped/ v34n1/art07.pdf 